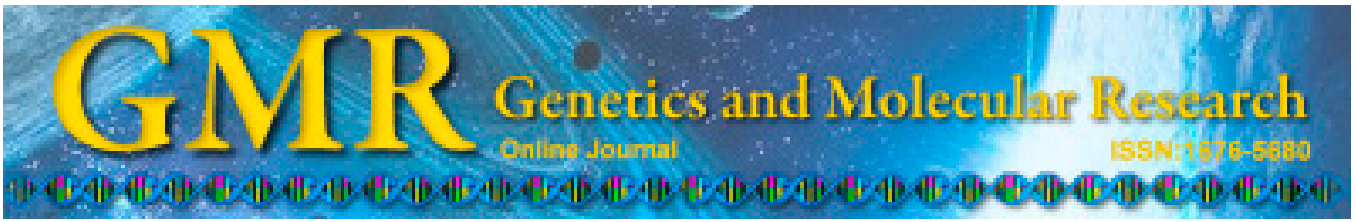

\title{
Local expression and role of BMP-2/4 in injured spinal cord
}

\author{
Z.S. Cui, P. Zhao, C.X. Jia, H.J. Liu, R. Qi, J.W. Cui, J.H. Cui, Q. Peng, \\ B. Lin and Y.J. Rao \\ Luoyang Orthopedic Hospital of Henan Province, Luoyang Henan, China \\ Corresponding author: Y.J. Rao \\ E-mail: yaojianrao@163.com
}

Genet. Mol. Res. 14 (3): 9109-9117 (2015)

Received January 7, 2015

Accepted May 8, 2015

Published August 7, 2015

DOI http://dx.doi.org/10.4238/2015.August.7.20

\begin{abstract}
We investigated local changes in BMP-2/4 expression in rat spinal cords 1 week following injury to study the damage effects of BMP-2/4 in spinal cord injury (SCI). Sprague Dawley rats $(45,4$ months old) were randomized into three groups comprising 15 rats each: a SHAM group, an SCI without noggin group (SCIO), and an SCI with noggin group (SCID). The SCIO and SCID groups were subjected to spinal cord hemisection, and motor activity was assessed using the BBB score. Expression of BMP-2/4 in each injured spinal cord section was examined by hematoxylin and eosin staining, immunohistochemistry, and western blot. There were no significant differences in BBB scores among the three groups $(\mathrm{P}>0.05)$. Following hemisection, the BBB score in the SHAM group was significantly higher than in the other two groups on the 1st day after modeling $(\mathrm{P}<0.05)$, and the BBB scores in the SCIO and SCID groups were not significantly different $(\mathrm{P}>0.05)$. Seven days after modeling, the BBB score in the SHAM group was significantly higher than in the other two groups $(\mathrm{P}<0.05)$, and the BBB score in the SCID group was obviously higher than in the SCIO group $(\mathrm{P}<0.05)$. The expression of $\mathrm{BMP}-2 / 4$ was highest in the SCIO group and lowest in the SHAM group $(\mathrm{P}<0.05)$. SCI can cause severe
\end{abstract}


impairment of motor activity in rats. Seven days after SCI, the local expression of BMP-2/4 had obviously increased; noggin can effectively inhibit the expression of BMP-2/4 and reduce impairment.

Key words: BMP-2/4; Noggin; Spinal cord injury

\section{INTRODUCTION}

Spinal cord injury (SCI) disrupts long-projection axons, with devastating neurological outcomes (Machado et al., 2008). Injured mammalian spinal cords and brains do not usually show spontaneous recovery, although neural precursor cells (NPCs) are present in adult spinal cords and brains. Adult NPCs proliferate in response to injury, yet the vast majority of newly generated cells become glial fibrillary acidic protein (GFAP)-positive astrocytes. When NPCs, either from the embryonic cerebral cortex or the adult subventricular zone, were grafted into lesioned adult rat spinal cord, the majority differentiated into cells with an astrocytic phenotype, and no neurons were detected (Tetzlaff et al., 2011). NPCs are multipotent progenitor cells present within the germinal zones of the central nervous system (CNS) throughout embryonic, postnatal, and adult life. They possess the ability to self-renew and generate different neural cell types within discrete temporospatial developmental windows when they encounter different signals (Katsimpardi et al., 2008; Zander et al., 2014). Recent studies have provided evidence that soluble protein mediators, such as cytokines and transcription factors, play critical roles in NPC fate determination. Previously, it was reported that when embryonic NPCs in culture were exposed to bone morphogenetic proteins (BMPs), a subgroup of the TGF- $\beta$ superfamily, their developmental fate was altered from neuronal to astrocytic cells. Undesirable gliogenesis after nerve injury and NPC transplantation may be due to the antineurogenic function of BMPs (Hao et al., 2003; Okano-Uchida et al., 2004; Zhang et al., 2014).

In the early development of the CNS, BMPs are expressed particularly at the lateral edges of the neural plate and subsequently in the dorsal midline of the neural tube, and regulate production of dorsal neural cells (Lü and $\mathrm{Hu}, 2009$ ). In adults, BMP-4 is widely expressed throughout the CNS, mainly in neurons and astrocytes (Cheng et al., 2007; Hampton et al., 2007). In general, NPCs can be isolated from the brain and spinal cord of embryonic, neonatal, or adult animals, and proliferated and differentiated under special conditions in vitro (Kitada et al., 2011). This provides a powerful tool for the study of lineage segregation of NPCs. In situ hybridization has indicated that expression of BMP-7 mRNA is present only in glial cells in uninjured spinal cord. After injury, the number of BMP-7-expressing glial cells is increased; BMP-7 expression also becomes apparent in motor neurons (Setoguchi et al., 2004). BMP-6 is expressed in neurons of the hippocampus and cortex in normal adult rat brains. A pronounced expression of BMP-6 in astroglia located at a lesion becomes obvious $48 \mathrm{~h}$ post-injury (Tesseur et al., 2006). These studies suggest that an inflammatory environment might influence the expression pattern of BMPs in the NPCs and their lineages.

Extracellular antagonists such as noggin can suppress the BMP signaling pathway by binding BMP ligands to prevent their association with BMP receptors (Liu and Niswander, 2005). In vitro experiments have demonstrated that BMPs mediate differentiation and maturation of astrocytes from adult mouse spinal cord-derived progenitor cells while suppressing both neuronal and oligodendroglial lineage (Setoguchi et al., 2004). In addition, BMPs pro- 
mote production of astrocytes from oligodendrocyte precursor cells (OPCs) at the expense of oligodendrocytes. Mabie et al. (1997) found that OPCs from postnatal rat cortex differentiated mainly into astrocytes instead of oligodendrocytes after BMP-2 stimulation. Since BMPs are involved in promoting astrogliogenesis at the late stage of CNS development, their expression levels are significant. There have been numerous in vitro studies on the effects of BMPs on cell proliferation and differentiation in many different cell types, including NPCs, OPCs, oligodendrocytes, and astrocytes. However, the expression patterns of BMPs and the effects of BMPs on the regulation of cell components in the injured CNS in vivo have not been determined. In this study, we characterized the temporal expression of BMP-2/4 and its relationship with motor activity in adult rat spinal cords following contusive injury.

\section{MATERIAL AND METHODS}

\section{Animals and reagents}

The Sprague Dawley rats (purchased from the SCXK Experimental Animal Center of Henan Province, China) were kept at the animal research facility of our institution. In addition, the experiments were performed according to the protocols of the University Animal Welfare and Ethics Review Committees. Antibodies for BMP-2 and BMP-4, and goat anti-mouse secondary antibody were purchased from the Wuhan Boster Biotechnology Co., Ltd. (Wuhan, China). Hematoxylin and eosin (H\&E) dye was purchased from the Nanjing Jiancheng Technology, Co., Ltd. (Nanjing, China). Noggin was purchased from PeproTech (Rocky Hill, NJ, USA).

\section{Animal modeling}

Sprague Dawley rats $(45,4$ months old) were randomly divided into three groups comprising 15 rats each: a sham-operated (SHAM) group; an SCI without noggin group (SCIO); and an SCI with noggin $(1 \mu \mathrm{g} / \mathrm{kg}$ ) group (SCID). All animals were anaesthetized by intraperitoneal injection of xylazine $(10 \mathrm{mg} / \mathrm{kg})$ and ketamine $(75 \mathrm{mg} / \mathrm{kg})$. Following back shaving and sterilization, an incision was made on the back posterior to the lower thoracic region. When the back muscles had been infiltrated, the dorsal surface of the spinal cord was exposed by laminectomy at the T10 level, and the lower thoracic cord was subsequently transected using fine scissors. Finally, the surgical wound was closed in two layers. All SHAM rats underwent a similar operation to those in the SCI group, except that the lower thoracic cord was exposed but not transected. SCI rats received daily assistance in bladder emptying until spontaneous miction recovered. All rats were fed with commercial rat chow available ad libitum comprising $0.95 \%$ calcium and $0.67 \%$ phosphate, and housed in a controlled environment at $22^{\circ} \mathrm{C}$ with a 12-h light/dark cycle. To prevent infection, $80,000 \mathrm{U}$ penicillin was injected intramuscularly each day for 7 days. If any rat died during modeling, a new rat was added to maintain the total number of 45 .

\section{Behavioral testing}

Basso, Beattie, and Bresnahan (BBB) scores were assigned the day before operation, the 1st day after operation, and the 7th day after operation (Basso et al., 1996). All rats 
were assigned randomly to ensure that the initial locomotor scores were equalized between groups. Severe compression injury produces spontaneous recovery to a BBB score of 4 . Behavioral testing was carried out daily using the BBB locomotor racing scale by two individuals "blinded" to the treatment of the rats. To elucidate whether these functional recoveries were due to axonal regeneration or activated local reflex of the segment below the injured site, we performed transections on all rats one segment distal (T10) from the previously injured spinal level. Transected rats were monitored for 7 days.

\section{Western blot}

Animals were sacrificed on the day before operation, on the 1st day after operation, and on the 7th day after operation. T10 spinal cord samples $(\mathrm{N}=3$ at each time point) were taken from the SHAM, SCIO, and SCID groups. Spinal cord samples were homogenized in $100 \mu \mathrm{L}$ lysis buffer with protease inhibitor with a dilution of 1:100 (Roche, Shanghai, China) and dithiothreitol ( $1 \mathrm{mg} / \mathrm{L} ; 1: 500)$. The lysate was sonicated three times ( $3 \mathrm{~s}$ each time) and centrifuged at $12,000 \mathrm{~g}$ for $25 \mathrm{~min}$ at $4^{\circ} \mathrm{C}$ to separate the protein into the supernatant. Protein concentrations were measured using a protein assay kit (BioRad, Philadelphia, PA, USA) and LabSystems Multiskan MS Plate Reader (LabSystems, Helsinki, Finland), and then 5X sample buffer was added, followed by heating the mixture for 8 min at $90^{\circ} \mathrm{C}$.

Equal amounts of protein extracts from each sample $(30 \mu \mathrm{g})$ were separated by sodium dodecyl sulfate-polyacrylamide gel electrophoresis on $10 \%$ polyacrylamide gel. Peptides were then electrophoretically transferred onto a polyvinylidene difluoride membrane (Millipore Corporation, Billerica, MA, USA) for $3 \mathrm{~h}$ at $400 \mathrm{~mA}$. Nonspecific binding was blocked by incubation for $1 \mathrm{~h}$ at room temperature in $10 \%$ dry milk diluted in Tris buffer saline, $\mathrm{pH} 7.5$, with $0.1 \%$ Tween-20 (TBST). Membranes were incubated overnight at $4{ }^{\circ} \mathrm{C}$ with mouse primary antibody against BMP-2 and BMP-4. All dilutions were made in TBST. Blots were rinsed in TBST three times for 10 min each and incubated for $1 \mathrm{~h}$ at room temperature with secondary horseradish peroxidase-conjugated anti-rabbit $\operatorname{IgG}(1: 1000)$ (Jinshan, Shanghai, China). Peptides were detected on a high-performance chemiluminescence film (GE Healthcare, New York, USA ) by an enhanced chemiluminescence Western blotting kit (GE Healthcare). Images were digitally scanned and analyzed with the ImageJ software (National Institutes of Health, Bethesda, MD, USA). Protein content was normalized according to $\beta$-actin content.

\section{Immunohistochemistry}

On the day before operation, the 1st day after operation, and the 7th day after operation, animals were killed by an overdose of pentobarbital sodium $(100 \mathrm{mg} / \mathrm{kg}$, ip $)$, followed by transcardial perfusion of $0.9 \%$ saline for $5 \mathrm{~min}$ and then $4 \%$ paraformaldehyde (Sigma, Shanghai, China) in $0.1 \mathrm{M}$ phosphate-buffered saline (PBS), pH 7.4, for $15 \mathrm{~min}$ at room temperature. Two $3-\mathrm{mm}$ spinal cord segments, which were $1.5 \mathrm{~mm}$ rostral and $1.5 \mathrm{~mm}$ caudal to the lesion epicenter, were removed and postfixed in the same fixative for $2 \mathrm{~h}$ at $4^{\circ} \mathrm{C}$ before being cryopreserved in $30 \%$ sucrose solution in PBS for $2-3$ days at $4^{\circ} \mathrm{C}$. Spinal cords were frozen in optimum cutting temperature compound (Tissue-Tek) and $15-\mu \mathrm{m}$ transverse sections were cut and mounted on gelatin-coated glass slides. Sections were preincubated in a blocking solution containing $8 \%$ normal donkey serum in the diluent solution $(1 \%$ bovine serum 
albumin and $0.3 \%$ Triton $\mathrm{X}-100$ in $0.01 \mathrm{M}$ PBS, $\mathrm{pH} 7.5$ ) for $1 \mathrm{~h}$ at room temperature. Sections were then incubated overnight at $4{ }^{\circ} \mathrm{C}$ with the primary antibodies in the diluent solution. Sections were then washed three times in $0.01 \mathrm{M}$ PBS for $10 \mathrm{~min}$ each and then incubated with the secondary antibodies diluted in $0.01 \mathrm{M}$ PBS for $1 \mathrm{~h}$ at room temperature in the dark. Sections were also counterstained with the nuclear dye. Negative controls consisted of secondary antibody alone. All samples were examined with a phase contrast microscope, and images were acquired using a digital camera and image-capturing software (Diagnostic Instruments Inc., Frankfurt, Germany).

\section{Statistical analysis}

The data were analyzed by one-way analysis of variance followed by Student-Newman-Keuls tests of multiple comparisons to determine whether there were significant differences between individual groups. All differences were considered to be significant at $\mathrm{P}<0.05$.

\section{RESULTS}

To investigate whether the modeling had been successful, we examined the BBB scores in the three groups (Table 1). There was no difference in the BBB score between the three groups before modeling. After the operation, the BBB scores in the three groups decreased to different extents. It should be noted that BBB scores in the SCI groups (SCIO and SCID groups) were significantly lower than in the SHAM group, which suggested that the SCI modeling was successful $(\mathrm{P}<0.05)$. Seven days later, the BBB scores in the three groups had recovered to different extents, especially in the SCID group (an 8-fold increase, $\mathrm{P}<0.05)$. The administration of noggin would alleviate the SCI to the CNS and relieve the symptoms.

Table 1. BBB scores in different time points $(\mathrm{N}=45)$.

\begin{tabular}{lcrr}
\hline Group & Before modeling & 1st day after modeling & 7th day after modeling \\
\hline SHAM group & $21.00 \pm 0.00$ & $18.38 \pm 0.42$ & $18.96 \pm 0.30$ \\
SCID group & $21.00 \pm 0.00$ & $1.54 \pm 0.55$ & $11.49 \pm 0.43$ \\
SCIO group & $21.00 \pm 0.00$ & $1.87 \pm 0.49$ & $4.09 \pm 0.44$ \\
\hline
\end{tabular}

To further verify the success of the modeling, H\&E staining of spinal cord sections was carried out. Under a high magnification (400X), the organizational structure of the spinal cord in the SHAM group was clear and there was no obvious cell damage (Figure 1). However, there were obvious disorders and loose arrangement of structure in the SCID and SCIO groups, and clear blank areas were visible, which suggested the formation of glial scars. The cell structures in the SCI groups were not complete, and the cells exhibited demyelinating change, neuron vacuole degeneration, and nuclear pyknosis. Moreover, there was a large number of polymorphonuclear cells and extensive macrophage infiltration. The SCIO group even exhibited edema and massive bleeding. Compared with the SCIO group, the structure in the SCID group was much more ordered and clear, and fewer necrosis areas and glial scars were observed. These results further verified the success of the SCI modeling and the effect of noggin in relieving SCI. 


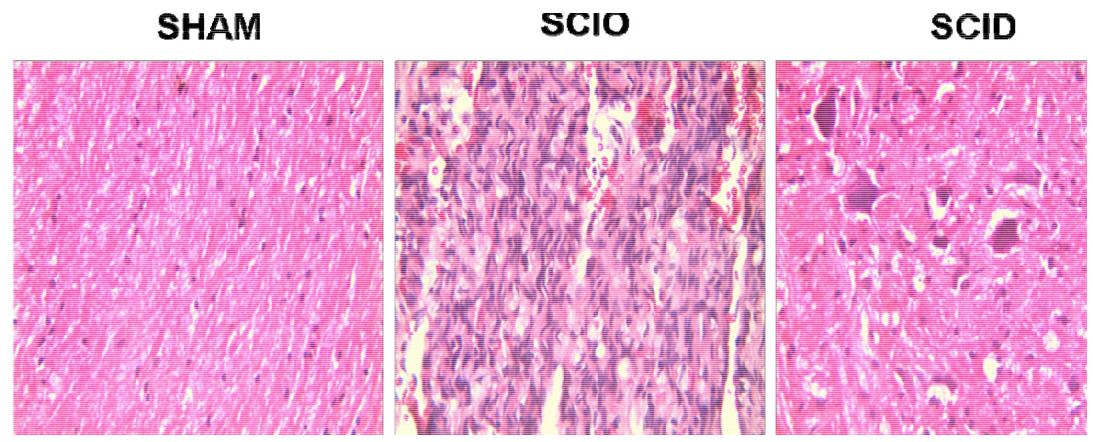

Figure 1. H\&E staining of spinal cord tissue in different groups at the 7th day after modeling (magnification $=$ $400 \mathrm{X})$. SHAM = sham-operated group; $\mathrm{SCIO}=$ spinal cord injury without noggin group; $\mathrm{SCID}=$ spinal cord injury with noggin group.

The goal of this study was to investigate the BMP expression profiling in SCI in vivo, so the distribution of BMP-2/4 was detected by immunohistochemistry. As shown in Figure 2, the BMP-2/4-positive cells were dyed lightest in color and were highly dispersed in the SHAM group. A stronger dyeing of BMP-2/4 was observed in the SCID group and the number of BMP-2/4-positive cells was higher than in the SHAM group. The strongest and most prevalent BMP-2/4 staining was seen in the SCIO group.

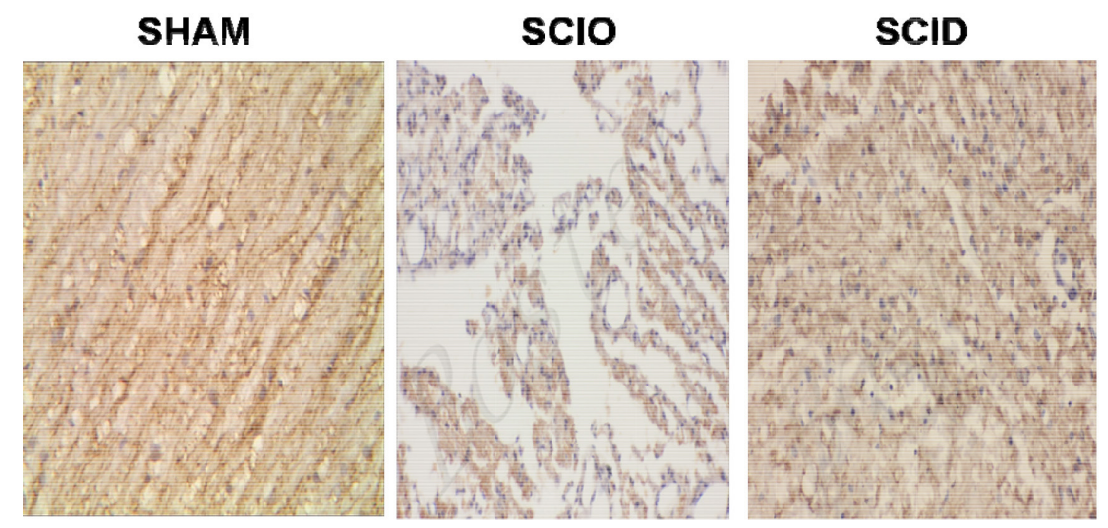

Figure 2. BMP 2/4 expression pattern in spinal cord in different groups at the 7 th day after modeling (magnification $=200 \mathrm{X})$. For abbreviations, see legend to Figure 1 .

The expression of BMP-2/4 was further detected by western blot (Figure 3). In the SHAM group, the expression of BMP-2/4 was lowest and a slight increase was seen on the 7 th day after operation. This was consistent with the previous finding that BMP-2/4 is not expressed in normal spinal cord (Setoguchi et al, 2004). The expression increased significantly (by more than 3 -fold) in the SCI groups compared with the SHAM group $(\mathrm{P}<0.05)$. Moreover, the expression level was obviously higher ( 1.5-fold) in the SCIO group than in the SCID group $(\mathrm{P}<0.05)$. These results show that SCI can cause an increased expression of BMP-2/4 and the effect can be inhibited by noggin injection. 
A

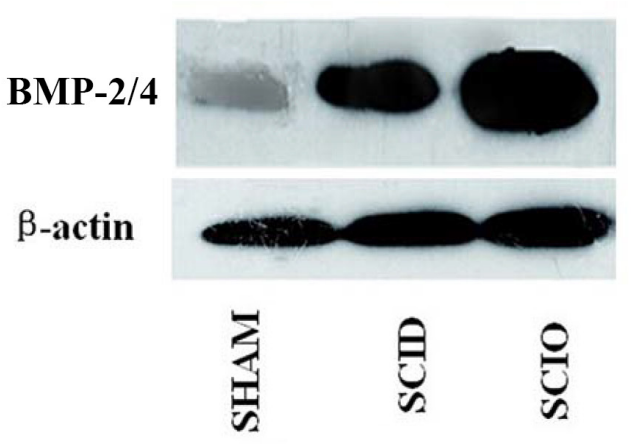

B

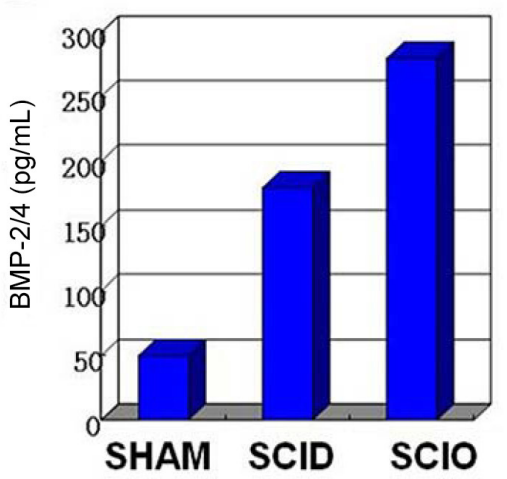

Figure 3. BMP 2/4 expression was determined by western blot (A) and ELISA (B) in different groups at the 7th day after modeling. For abbreviations, see legend to Figure 1.

\section{DISCUSSION}

Axon demyelination and glial scar formation are among the most important pathological changes following SCI. Therefore, promoting myelin sheath formation around the remaining axons and inhibiting glial scars are of great significance for the improvement of nerve function following SCI (Kopp et al., 2010). Oligodendrocytes are the only source in the CNS of sheath cells, which are structurally constituted by the expression of myelin basic protein and myelin lipoprotein (Gaultier et al., 2009). Therefore, promoting the formation and differentiation of oligodendrocytes following SCI is the only way to promote axon and myelin sheath formation. Astrocytes are activated and proliferate quickly following SCI, and the proliferated astrocytes constitute the physical component of glial scars (Li et al., 2010). In addition, the activated astrocytes can secrete GFAP, which constitutes the chemical component of glial scars (Zhao et al., 2004). These two components make it impossible for the axons to penetrate the glial scar, and the growth of the axons is blocked. Glial scars can also release axon growth inhibitory factors, such as chondroitin sulfate proteoglycan (Jeong et al., 2012). Therefore, it is important to inhibit the formation of glial scars and promote the growth of axons by inhibiting the activation and proliferation of astrocytes.

BMP, a subgroup of the TGF- $\beta$ superfamily, is a type of extracellular ligand (Hendy et al., 2005). It was initially isolated from decalcified bone matrix extract, and can induce the directional differentiation of undifferentiated mesenchymal cells into osteoblasts and form calcified osseous tissue, hence its name (Gazzerro et al., 1998). In recent years, studies have shown that BMP plays an important role in the development of the CNS and the regulation of cell fate, proliferation, and differentiation at different stages of neural development and in different areas of the CNS (Chen and Panchision, 2007; Ille et al., 2007). There are numerous members of the BMP family and they have different functions; they include BMP-2, -4 , and -7 , which can inhibit the generation of neuron oligodendrocytes, and induce the formation of type II astrocytes (Li et al., 2011).

The mechanisms by which BMPs act in neuron development are mainly as follows. BMPs act on cells by autocrine and paracrine signaling. In extracellular signaling, BMP com- 
bines with and activates specific receptors on the target cell membrane. The signals are then transduced in two ways: the Smad-dependent pathway and the Smad-independent pathway. In the Smad-dependent pathway, BMP combines with type I and type II receptors in the outer membrane of OPCs; type II receptors can activate type I receptors by phosphorylation. The phosphorylated type I receptors further activate the downstream effector protein to phosphorylate Smad producing R-Smad, which is a type of intracellular signal molecule, leading to signal transduction. The phosphorylated R-Smad is activated and released from the receptor to form a hetero-oligomeric complex co-Smad (such as Smad-4). Common R-Smad proteins include Smad-1, $-2,-3,-5$, and -8 . The complex can be activated by a transcription factor (transcriptional coactivator, P300) to form an Smad/P300 compound, which can enter the nucleus and activate the promoter of GFAP, inducing the differentiation of OPCs to astrocytes (Parikh et al., 2011).

In addition, BMP can upregulate the expression of DNA-binding inhibitory factor 4 (ID4) in OPCs to downregulate the expression of oligodendrocyte transcription factor 2 (OLIG2), as ID4 is a binding protein for OLIG2. ID4 can combine with OLIG2 in the cell plasma and block the nuclear transfer of OLIG2, thereby further inhibiting the differentiation of OPCs to oligodendrocytes. Moreover, the blocked OLIG2 can bind to P300 in the cell plasma of OPCs, inhibit the formation of the Smad/P300 compound, and inhibit the activation of GFAP. This inhibition effect blocks the differentiation of OPCs to astrocytes (Cheng et al., 2007). Setoguchi et al (2004) found that there was no expression of BMP-2/4 in normal spinal cord, and expression of BMP-7 was only observed in anterior horn motor neurons and oligodendrocytes. After SCI, the expression of BMP is decreased in oligodendrocytes and increased in astrocytes, which suggests that BMP plays different roles in these two types of cells, although the specific mechanism requires further investigation. OPCs are the main source of oligodendrocytes. Current studies have confirmed that there is an OPC pool in the adult CNS, and OPCs have pluripotent potential to differentiate into oligodendrocytes and type II astrocytes. After SCI, some astrocytes are activated and proliferate with increased expression of BMP-2/4, which can further stimulate the differentiation of OPCs into type II astrocytes and inhibit their differentiation into oligodendrocytes.

\section{CONCLUSIONS}

In this study, we proved that the local expression of BMP-2/4 has increased dramatically by the 7th day following SCI, and intervention with noggin can inhibit this expression pattern. When the expression of BMP-2/4 is inhibited, differentiation of OPCs into type II astrocytes can change to differentiation into oligodendrocytes. This change not only promotes the myelination of the remaining axons and inhibits the formation of glial scars, but also promotes the growth and connection of axons. Therefore, research into a means of inhibiting BMP-2/4 expression would be valuable for the treatment of SCI.

\section{Conflicts of interest}

The authors declare no conflict of interest. 


\section{REFERENCES}

Basso DM, Beattie MS and Bresnahan JC (1996). Graded histological and locomotor outcomes after spinal cord contusion using the NYU weight-drop device versus transection. Exp. Neurol. 139: 244-256.

Chen HL and Panchision DM (2007). Concise review: bone morphogenetic protein pleiotropism in neural stem cells and their derivatives - alternative pathways, convergent signals. Stem Cells 25: 63-68.

Cheng X, Wang Y, He Q, Qiu M, et al. (2007). Bone morphogenetic protein signaling and olig1/2 interact to regulate the differentiation and maturation of adult oligodendrocyte precursor cells. Stem Cells 25: 3204-3214.

Gaultier A, Wu X, Le Moan N, Takimoto S, et al (2009). Low-density lipoprotein receptor-related protein 1 is an essential receptor for myelin phagocytosis. J. Cell Sci. 122: 1155-1162.

Gazzerro E, Gangji V and Canalis E (1998). Bone morphogenetic proteins induce the expression of noggin, which limits their activity in cultured rat osteoblasts. J. Clin. Invest. 102: 2106-2114.

Hampton DW, Asher RA, Kondo T, Steeves JD, et al. (2007). A potential role for bone morphogenetic protein signalling in glial cell fate determination following adult central nervous system injury in vivo. Eur. J. Neurosci. 26: 3024-3035.

Hao HN, Zhao J, Thomas RL, Parker GC, et al. (2003). Fetal human hematopoietic stem cells can differentiate sequentially into neural stem cells and then astrocytes in vitro. J. Hematother. Stem Cell Res. 12: 23-32.

Hendy GN, Kaji H, Sowa H, Lebrun JJ, et al. (2005). Menin and TGF-beta superfamily member signaling via the Smad pathway in pituitary, parathyroid and osteoblast. Horm. Metab. Res. 37: 375-379.

Ille F, Atanasoski S, Falk S, Ittner LM, et al. (2007). Wnt/BMP signal integration regulates the balance between proliferation and differentiation of neuroepithelial cells in the dorsal spinal cord. Dev. Biol. 304: 394-408.

Jeong SR, Kwon MJ, Lee HG, Joe EH, et al. (2012). Hepatocyte growth factor reduces astrocytic scar formation and promotes axonal growth beyond glial scars after spinal cord injury. Exp. Neurol. 233: 312-322.

Katsimpardi L, Gaitanou M, Malnou CE, Lledo PM, et al. (2008). BM88/Cend1 expression levels are critical for proliferation and differentiation of subventricular zone-derived neural precursor cells. Stem Cells 26: 1796-1807.

Kitada M, Kuroda Y and Dezawa M (2011). Lectins as a tool for detecting neural stem/progenitor cells in the adult mouse brain. Anat. Rec. 294: 305-321.

Kopp MA, Brommer B, Gatzemeier N, Schwab JM, et al. (2010). Spinal cord injury induces differential expression of the profibrotic semaphorin 7A in the developing and mature glial scar. Glia 58: 1748-1756.

Li HF, Jiang XH, Zou DQ, Cao QL, et al. (2011). Expression of bone morphogenetic protein receptor IA in rats after contusive spinal cord injury. Nan Fang Yi Ke Da Xue Bao 31: 1124-1130.

Li Z, Fang ZY, Xiong L and Huang XL (2010). Spinal cord injury-induced astrocyte migration and glial scar formation: effects of magnetic stimulation frequency. Indian J. Biochem. Biophys. 47: 359-363.

Liu A and Niswander LA (2005). Bone morphogenetic protein signalling and vertebrate nervous system development. Nat. Rev. Neurosci. 6: 945-954.

Lü HZ and Hu JG (2009). Expression of bone morphogenetic proteins-2/4 in neural stem cells and their lineages. Acta Neurobiol. Exp. 69: 441-447.

Mabie PC, Mehler MF, Marmur R, Papavasiliou A, et al. (1997). Bone morphogenetic proteins induce astroglial differentiation of oligodendroglial-astroglial progenitor cells. J. Neurosci. 17: 4112-4120.

Machado P, Gawronski J and Gall A (2008). Ankylosing spondylitis and spinal cord injury. Acta Reumatol. Port. 33: 231-237.

Okano-Uchida T, Himi T, Komiya Y and Ishizaki Y (2004). Cerebellar granule cell precursors can differentiate into astroglial cells. Proc. Natl. Acad. Sci. 101: 1211-1216.

Parikh P, Hao Y, Hosseinkhani M, Patil SB, et al. (2011). Regeneration of axons in injured spinal cord by activation of bone morphogenetic protein/Smad1 signaling pathway in adult neurons. Proc. Natl. Acad. Sci. 108: E99-E107.

Setoguchi T, Nakashima K, Takizawa T, Yanagisawa M, et al. (2004). Treatment of spinal cord injury by transplantation of fetal neural precursor cells engineered to express BMP inhibitor. Exp. Neurol. 189: 33-44.

Tesseur I, Zou K, Berber E, Zhang H, et al. (2006). Highly sensitive and specific bioassay for measuring bioactive TGFbeta. BMC Cell Biol. 7: 15.

Tetzlaff W, Okon EB, Karimi-Abdolrezaee S, Hill CE, et al. (2011). A systematic review of cellular transplantation therapies for spinal cord injury. J. Neurotrauma 28: 1611-1682.

Zander MA, Cancino GI, Gridley T, Kaplan DR, et al. (2014). The Snail transcription factor regulates the numbers of neural precursor cells and newborn neurons throughout mammalian life. PLoS One 9: e104767.

Zhang Y, Qi XM, Yao YM, Liu ZH, et al. (2014). Expression pattern of transcription factor SOX2 in reprogrammed oligodendrocyte precursor cells and microglias: Implications for glial neurogenesis. Acta Neurobiol. Exp. 74: 33-43.

Zhao Z, Alam S, Oppenheim RW, Prevette DM, et al. (2004). Overexpression of glial cell line-derived neurotrophic factor in the CNS rescues motoneurons from programmed cell death and promotes their long-term survival following axotomy. Exp. Neurol. 190: 356-372. 